\title{
A SIMPLE MHD MODEL FOR ONE-SIDED JETS
}

\author{
G. BODO, E. TRUSSONI \\ Osservatorio Astronomico di Torino, Italy
}

\section{G. CHAGELISHVILI}

Abastumani Astrophysical Obervatory, Georgian Republic

The one-sideness observed in several extragalactic jets is usually explained by the Doppler boosting effect in a pair of relativistic beams, moving almost parallel to the line of sight. However jets could be intrinsically asymmetric if magnetically driven from the accretion disk surrounding the central black hole. Wang et al. (1992) have shown that a combination of odd and even magnetic structures could lead to different Poynting fluxes in the two hemispheres of the inner nucleus. However, without any peculiar assumption, it can be shown that intrinsic onesideness can be simply related to the symmetry properties of the MHD equations.

The magnetic configuration in the acceleration region can derive its poloidal component $B_{p}$ from a primordial large scale magnetic field, convected into the central region. This poloidal component is antisymmetric with respect to the equatorial plane of the disk. The disk differential (along the vertical direction) rotation leads to the formation of a toroidal magnetic field $\left(B_{t}\right)$, which changes sign across the equator (antisymmetric). On the other hand, the development of a turbulent dynamo process (Chagelishvili et al. 1990) can originate a further toroidal magnetic component $\left(B_{t, d}\right)$ in the disk, much larger than $B_{t}$ and symmetric with respect to the equatorial plane.

Outflow solutions in standard MHD models (Camenzind 1989) usually make use of an antisymmetric toroidal component of the magnetic field. As the MHD equations are invariant by changing the sign of both velocity and magnetic field, in this case $\left(B_{t, d}=0\right)$ we expect symmetric acceleration (i.e. double jets) from both sides of the equator. If conversely the dynamo works $\left(B_{t, d} \neq 0\right)$, in one hemisphere the same previous outflow solution is found. On the other side of the disk outflowing solutions are also found but, as can be seen from the Bernoulli equation, either the flow in not magnetically driven, or the Poynting flux is opposite to the outflow velocity. In the former case a new acceleration process must be proposed, while in the second case we can expect the solution to be unstable.

\section{References}

Camenzind M. 1989, in Accretion Disks and Magnetic Fields in Astrophysics, ed. G. Belvedere (Dordrecht:Kluwer), 129

Chagelishvili G. et al. 1990, in Plasma Astrophysics, ESA SP-311, 273

Wang J.C.L., Sulkanen M.E. \& Lovelace R.V.E. 1992, ApJ 390, 46 